\section{A persistent problem}

The phenomenon of bacterial persistence, the existence in a bacterial population of dormant cells that are tolerant to killing by many bactericidal antibiotics, is thought to be an important contributor to the failure by antibiotics to eradicate chronic infections (Mulcahy et al., 2010). This may be particularly important with biofilmforming organisms, such as Pseudomonas aeruginosa, since the fraction of persister cells is much higher in biofilms than during planktonic growth (Spoering \& Lewis, 2001). Similarly, there is a higher level of persistence among stationary (as much as $1 \%)$ than exponential growth $\left(10^{-5}\right.$ or less $)$ (Keren et al., 2004a). Persisters are phenotypic variants of normal cells, as they are not genetically different from their nonpersistent siblings. The mechanisms regulating the level of persistence in a population are unclear (Lewis, 2007), as discussed further below. It has been proposed that agents which decrease or eliminate persistence (or persisters) could be used as adjunct therapy along with standard antibiotics (Smith \& Romesberg, 2007) in order to better treat recalcitrant infections - but this presupposes that specific functions responsible for persister formation can be identified.

An article in the present issue of the Journal of Medical Microbiology (De Groote et al., 2011) appears to implicate a small molecule effector as having a role in persister formation in $P$. aeruginosa. This interesting finding arose from studies of a mutant exhibiting reduced persister formation (as measured by the fraction of survivors of ofloxacin treatment during stationary phase) isolated in a high throughput screen of a mutant library of $P$. aeruginosa. The mutant contained a plasposon insertion in gene PA14_4970 but was found to lead to overexpression of a downstream gene, fos $A$, a gene known to encode a glutathione transferase that can inactivate the antibiotic fosfomycin. Overexpression of FosA was found to be responsible for both the reduced persister formation and fosfomycin resistance and these both required FosA enzymic activity. The magnitude of the effect on persistence was not great (two- to fivefold depending upon the strain used) but was reproducible. Exploring further, the authors found that a $g l p T$ mutation [transporter of glycerol 3-phosphate (G3P)], which causes fosfomycin resistance by preventing its transport, had an effect on persister formation similar to that of FosA. The correlation of fosfomycin resistance and lowered persistence led to the proposal of a model in which an organic phosphate or phosphonate that is a substrate of both GlpT transport and FosA glutathione conjugation (as is fosfomycin) can act as a positive regulator of persistence. Thus, those functions which prevent fosfomycin from its antibiotic action would prevent this unknown organic phosph[on]ate from promoting persistence. The authors note, as well, the crosstalk of fosfomycin resistance and persistence and that, while in this case fosfomycin resistance leads to decreased persistence, the possibility of interplay between resistance and persistence should be further studied if treatment with combinations of antibiotics and persistence inhibitors is contemplated. Some comments and speculation on recent findings regarding persistence may provide further context for this intriguing work.

In the effort to define players in the production of persisters, a number of studies have detected gene products which when upregulated lead to increased persistence, generally defined as tolerance to challenge with specific antibiotics. Several of these have been identified as toxin-encoding members of toxin/ antitoxin (TA) 'addiction' modules (Lewis, 2010). The toxins can induce reversible dormancy by inhibiting specific areas of metabolism. A transcriptome analysis of persisters isolated as survivors after ampicillin lysis showed upregulation of a number of TA modules and other functions that can inhibit translation, DNA replication and cell division (among others) (Keren et al., 2004b). Thus, persistence may be viewed as a state in which a subpopulation of cells becomes tolerant to bactericidal antibiotics because the processes that they target are turned off. It should be noted that there have been no TA modules identified in $P$. aeruginosa.

Identification of genes whose deletion leads to elimination of persistence, and hence are necessary for the production of persisters (and might be targets for adjunct therapy), has been less fruitful. In a screen of a library of ordered in-frame knockouts of non-essential genes in Escherichia coli, inactivation of a number of global regulators and two genes involved in nucleotide metabolism was found to lower persistence levels but not to affect antibiotic MICs; however, none of these had more than a tenfold effect on persister production (Hansen et al., 2008). The lack of detection of deletions that completely eliminate persisters has led to the idea that there are likely to be redundant processes that can lead to persistence (Lewis, 2010).

Indeed, there appear to be a variety of pathways which, depending on growth conditions and ambient stress, can promote persistence. An important recent study by Fung et al. (2010) demonstrates the complexity of factors that can lead to antibiotic tolerance. This work determined the degree of tolerance (persistence) to treatment by different antibiotics as a function of growth medium, growth rate, starvation for particular nutrients singly and in combination, treatment with translation inhibitors, and bacteriostatic agents; additionally, the effect of a variety of gene deletions reported to reduce persistence was evaluated under a variety of growth conditions. The results were, unsurprisingly, complex. There is no simple course to persistence or tolerance. A variety of combinations of factors may lead to tolerance to one or more antibiotics, with certain pathways predominating under specific conditions. There may well be underlying mechanisms that play a broad role and would be suitable targets for a 
universal persistence inhibitor, but no unequivocal candidates have yet appeared.

Nevertheless, genetic approaches have turned up some interesting leads. As De Groote et al. (2011) note, their finding implicating an organic phosph[on]ate in persistence formation may be related to the observation that deletion of the $E$. coli gene $g l p D$, encoding sn-glycerol-3-phosphate dehydrogenase, leads to a reduction in persistence (Spoering et al., 2006). In the glpD deletion strain, it appears that high levels of G3P (caused by lack of catabolism by GlpD) plus derepression of the Glp regulon (which is induced by G3P interacting with the GlpR repressor of the regulon) lead to suppression of the formation or maintenance of persisters. Perhaps the putative organic phosph[on]ate promoter of persistence does so by competing with G3P in its role of reducing persistence, for example by preventing its interaction with GlpR. However, Spoering et al. (2006) found that, in E. coli, deletion of $g l p T$ (which is part of the Glp regulon) does not reduce persistence (Spoering et al., 2006) as it does in $P$. aeruginosa (De Groote et al., 2011). But this may be explained by the fact that, in E. coli, there are several possible transporters for G3P, with Ugp being the main transporter for G3P under phosphate starvation conditions (Xavier et al., 1995), while in P. aeruginosa, GlpT is likely to be the only transporter for G3P, as no orthologues for $u g p A$ or $u g p B$ are found in $P$. aeruginosa. It would be of interest to test the effects on persistence of deletions in $g l p T$ and $g l p D$ in a $\mathrm{Ugp}^{-}$background in E. coli.

While it is true that crosstalk between antibiotic resistance and persistence had not been reported previously to the observation of De Groote et al. (2011), it has been recently demonstrated that, in $E$. coli, treatment with fluoroquinolones not only selects for persisters but specifically induces their formation (Dörr et al., 2009). This is mediated through a DNA damageinduced SOS regulon-regulated toxin (TisB) (Dörr et al., 2010). Thus, to understand clinical responses to antibiotics as well as to design new strategies for treatment of chronic infections, it is important that the crosstalk among resistance, persistence and antibiotic treatment itself be further explored.

\section{Lynn L. Silver}

LL Silver Consulting LLC, 955 S. Springfield Avenue, Unit C403, Springfield, NJ 07081, USA

\section{Correspondence: Lynn L. Silver (silverly@comcast.net)}

De Groote, V. N., Fauvart, M., Kint, C. I., Verstraeten, N., Jans, A., Cornelis, P. \&

Michiels, J. (2011). Pseudomonas aeruginosa fosfomycin resistance mechanisms affect noninherited fluoroquinolone tolerance. $\mathrm{J} \mathrm{Med}$ Microbiol 60, 329-336.

Dörr, T., Lewis, K. \& Vulić, M. (2009). SOS response induces persistence to fluoroquinolones in Escherichia coli. PLoS Genet 5, e1000760.

Dörr, T., Vulić, M. \& Lewis, K. (2010)

Ciprofloxacin causes persister formation by inducing the TisB toxin in Escherichia coli. PLoS Biol 8, e1000317.

Fung, D. K. C., Chan, E. W. C., Chin, M. L. \& Chan, R. C. Y. (2010). Delineation of a bacterial starvation stress response network which can mediate antibiotic tolerance development. Antimicrob Agents Chemother 54, 1082-1093.

Hansen, S., Lewis, K. \& Vulić, M. (2008). Role of global regulators and nucleotide metabolism in antibiotic tolerance in Escherichia coli. Antimicrob Agents Chemother 52, 2718-2726.

Keren, I., Kaldalu, N., Spoering, A., Wang, Y. \& Lewis, K. (2004a). Persister cells and tolerance to antimicrobials. FEMS Microbiol Lett 230, 13-18.

Keren, I., Shah, D., Spoering, A., Kaldalu, N. \& Lewis, K. (2004b). Specialized persister cells and the mechanism of multidrug tolerance in Escherichia coli. J Bacteriol 186, 8172-8180.

Lewis, K. (2007). Persister cells, dormancy and infectious disease. Nat Rev Microbiol 5, 48-56.

Lewis, K. (2010). Persister cells. Annu Rev Microbiol 64, 357-372.

Mulcahy, L. R., Burns, J. L., Lory, S. \& Lewis, K. (2010). Emergence of Pseudomonas aeruginosa strains producing high levels of persister cells in patients with cystic fibrosis. J Bacteriol 192, 6191-6199.

Smith, P. A. \& Romesberg, F. E. (2007). Combating bacteria and drug resistance by inhibiting mechanisms of persistence and adaptation. Nat Chem Biol 3, 549-556.

Spoering, A. L. \& Lewis, K. (2001). Biofilms and planktonic cells of Pseudomonas aeruginosa have similar resistance to killing by antimicrobials. J Bacteriol 183, 6746-6751.

Spoering, A. L., Vulić, M. \& Lewis, K. (2006). GlpD and PlsB participate in persister cell formation in Escherichia coli. J Bacteriol 188, 5136-5144.

avier, K. B., Kossmann, M., Santos, H. \& Boos, W. (1995). Kinetic analysis by in vivo ${ }^{31} \mathrm{P}$ nuclear magnetic resonance of internal $\mathrm{P}_{\mathrm{i}}$ during the uptake of $s n$-glycerol-3-phosphate by the pho regulon-dependent Ugp system and the $g l p$ regulon-dependent GlpT system. J Bacteriol 177, 699-704. 